\title{
Focused Ion Beam Tomography of Porous Titania Thin Films
}

\author{
D. Vick ${ }^{1}$, K.M. Krause ${ }^{2}$, M.J. Brett ${ }^{1,2}$ \\ ${ }^{1}$ National Institute for Nanotechnology, 11421 Saskatchewan Dr., Edmonton, Alberta, Canada T6G 2M9. \\ 2 Department of Electrical and Computer Engineering, University of Alberta, Edmonton, Alberta, Canada \\ T6G 2 G7.
}

The technique of focused ion beam (FIB) tomography has witnessed rapid development in recent years [1]. Unlike traditional tomography, in which a sample is imaged successively from different orientations, FIB tomography involves the serial slicing and imaging of parallel surfaces, followed by a $3 \mathrm{D}$ reconstruction of the milled volume by means of post-processing software. A number of challenges associated with this technique have been identified, and significant advancement has been made in addressing them [2-4]. The issues include precision of the slicing increment, proper planarization of the milled surfaces, differential sputtering effects, imaging probe penetration depth, and ion-induced damage of the specimen. As FIB tomography approaches the nanoscale, the importance of these issues only increases.

We report on current work on the milling and imaging of nanostructured heterogeneous specimens aimed at investigating some of these issues. Our test specimens are porous thin films comprised of $\mathrm{TiO}_{2}$, embedded in a matrix of HPR 504 photoresist (PR) to form a heterogeneous solid. The thin films are created by means of electron beam evaporation using the Glancing Angle Deposition (GLAD) technique [5]. The films are next subjected to a PR spin step for infiltration of pores [6]. A number of features of the specimens make them suitable for FIB tomography: (i) they exhibit good atomic number $(\mathrm{Z})$ contrast, allowing resolution testing of backscattered-electron images; (ii) the fraction of voiding in the sample can be kept small, thus reducing the difficulties that are associated with 3D interpretation of porous specimens; (iii) the PR layer can be made thicker than the original porous film, thus eliminating the need to lay down a protective layer prior to the sectioning step; and (iv) the detail in the columnar structures of the original film range over scale lengths from 5 to several hundred nanometers, permitting one to assess the resolution limits of the 3D reconstruction. Figure 1 shows a SEM image of view of surface of one of our samples, freshly exposed by the FIB. This image was captured using with the Inlens SE detector at an electron high tension voltage setting of $1.0 \mathrm{kV}$.

A Zeiss NVision 40 Crossbeam ${ }^{\circledR}$ Workstation is used to perform the sequential slicing and imaging. In order to reconstruct the milled volumes and recover the nanostructure of the GLAD films, MATLAB $^{\mathrm{TM}}$ functions have been written to perform drift alignment of image sets. The commercially available JEOL TEMography ${ }^{\mathrm{TM}}$ software packages are used to render and interpret the reconstructed volumes [7]. The milled volumes of interest range from $100 \mathrm{um}^{3}$ down to $5 \mathrm{um}^{3}$. One of the aims of the study is optimization the imaging probe conditions, which will necessarily be a compromise between image probe size, that decreases with incident electron energy, and penetration depth, that increases with incident electron energy. As an additional aid in assessing the resolution limits of the tomographic reconstruction, we have at our disposal STEM and TEM capability that can be used in conjunction with the FIB to extend the resolution below that achievable by the FIB. 
The data obtained from the FIB provide insight into the porosity and growth scaling behaviour of GLAD films. Slice sequences aligned parallel to the Si substrates are most appropriate for this, as exemplified in Figure 2, where a freshly exposed layer of $\mathrm{TiO}_{2} / \mathrm{PR}$ is shown, again imaged with the Inlens detector at an electron high tension voltage of $1.0 \mathrm{kV}$. The curtaining striations visible in the top third of the micrograph were created a during a preparatory rough milling step performed at right angles to the sequence milling steps, and were caused by the differential sputtering rates of the oxide and PR. The curtaining effect must be minimized in the exposed section faces by a suitable choice of milling parameters. Once a series of images is obtained, post processing software may be used to assess porosity and collect statistics on the size distribution the columns.

\section{References}

[1] G. Möbus, B.J. Inkson, Materials Today 10(12), 18 (2007).

[2] A.J. Kubis, G.J. Shiflet, D.N. Dunn, R. Hull, Metall. Mater. Trans. A 35, 1935 (2004).

[3] A.J. Kubis, T.E. Vandervelde, J.C. Bean, D.N. Dunn, R. Hull, Appl. Phys. Lett. 88, 263103 (2006).

[4] R.K. Bansal, A. Kubis, R. Hull, J.M. Fitz-Gerald, J. Vac. Sci. Technol. B 24(2), 554 (2006).

[5] K. Robbie, M.J. Brett, A. Lakhtakia, Nature 384, 616 (1996).

[6] K.D. Harris, K.L. Westra, M.J. Brett, Electrochem. Solid-State Lett. 4(6), C39 (2001).

[7] www.temography.com

[8] The authors wish to acknowledge NINT and NSERC for financial support and the Canada Foundation for Innovation for funding the purchase of the NVision 40 microscope.

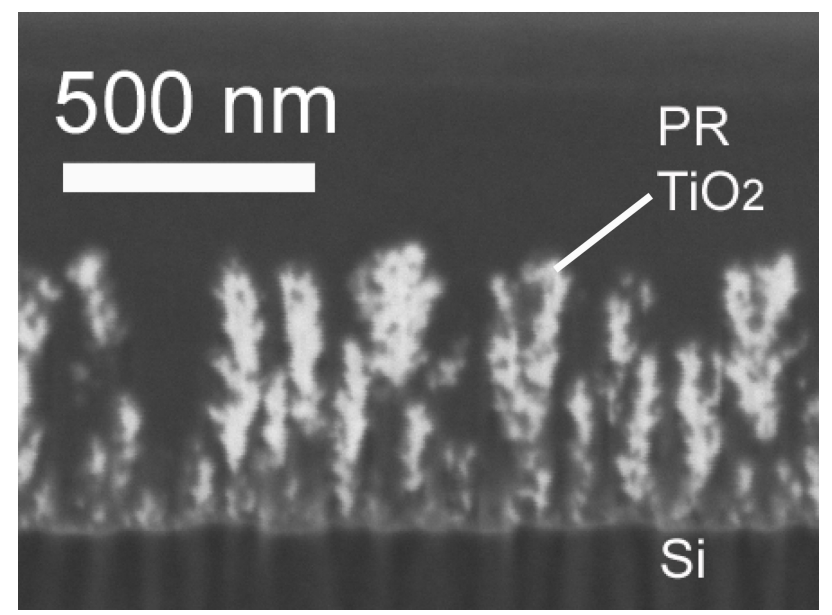

Figure 1: Inlens SEM image of a heterogeneous sample consisting of $\mathrm{TiO}_{2}$ embedded in polymer photoresist. This edge view was captured immediately after a FIB milling slice oriented normal to the substrate surface.

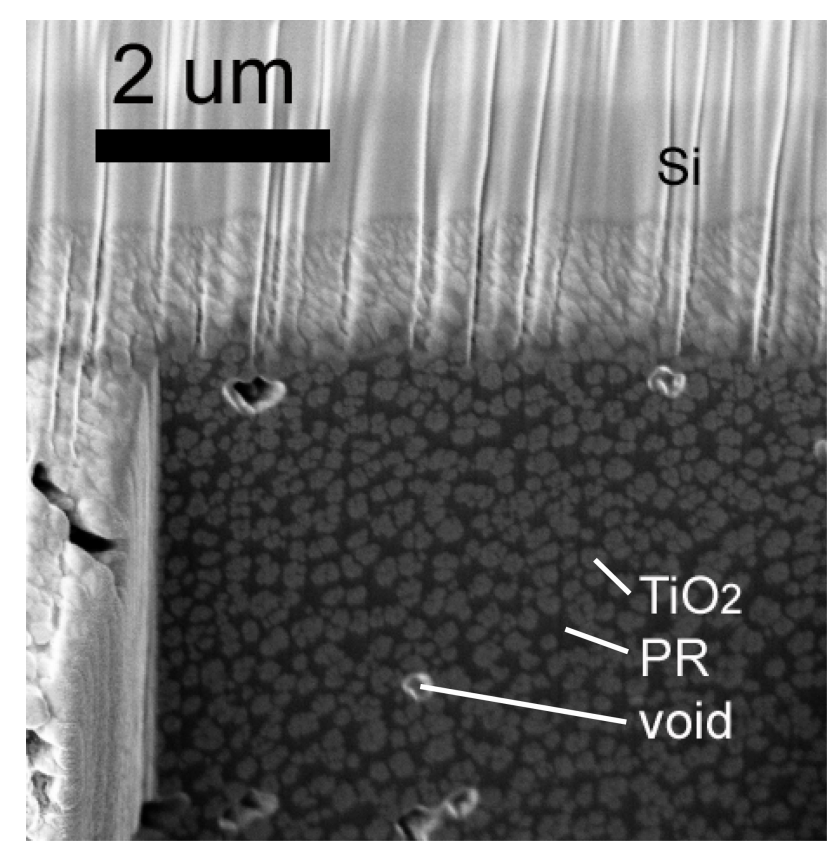

Figure 2: Inlens SEM image of an FIB exposed layer, cut parallel to the Si substrate and approximately $700 \mathrm{~nm}$ from the coating/substrate interface. 